\title{
Heidi Heilmann
}

\section{Professorin für Wirtschaftsinformatik und Mitbegründerin der HMD - Praxis der Wirtschaftsinformatik}

\author{
Matthias Knoll (D)
}

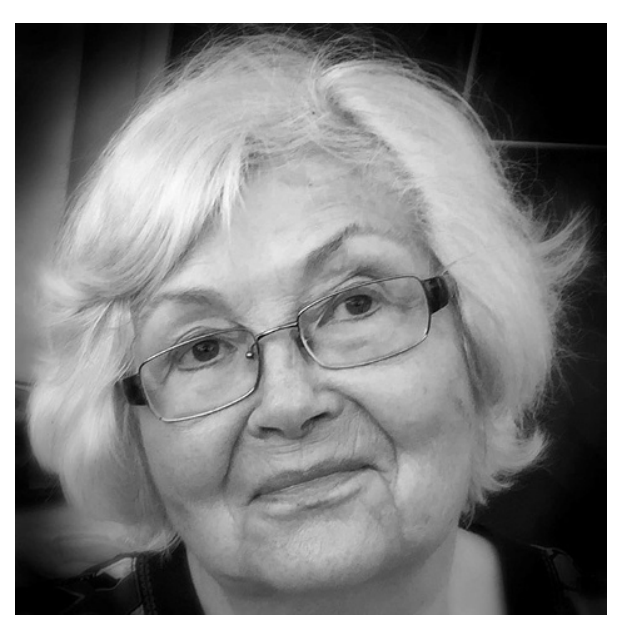

Dass das, was wir heute als leistungsstarke, vernetzte Informationstechnologie kennen, sich so schnell entwickeln und für unseren beruflichen wie privaten Alltag so überaus große Bedeutung erlangen würde, ahnte Heidi Heilmann vermutlich selbst nicht, als sie in den 1950er-Jahren ihr Studium der Volkswirtschaftslehre in Stuttgart und Tübingen begann.

Doch während ihren ersten Begegnungen von 1960 bis 1965 bei IBM Deutschland mit der IT in Form konventioneller Lochkartenmaschinen und „frühen“ Computern, deren Rechenleistung heute von jedem Taschenrechner um ein Vielfaches übertroffen wird, erkannte sie schnell, dass diese ersten Rechenmaschinen, die im zivilen Bereich zunächst schwerpunktmäßig in der Lohn- und Gehaltsabrechnung großer Unternehmen eingesetzt wurden, das Potenzial aufwiesen, die Arbeit in den Fachabteilungen nicht nur zu optimieren, sondern grundlegend zu verändern.

Sicherlich war diese Erkenntnis eine wesentliche Motivation, von 1965 bis 1980 als geschäftsführende Gesellschafterin der Integrata GmbH (später AG) in Tübingen, bis 1998 dann als Anteilseignerin in ihrer Funktion als Mitglied des Aufsichts-

M. Knoll ( $₫)$

Hochschule Darmstadt, Darmstadt, Deutschland

E-Mail: matthias.knoll@h-da.de 
rats, eine zentrale Führungs- und Gestaltungsposition einzunehmen und zunächst haupt-, später nebenberuflich daran mitzuwirken, dass die Integrata AG rasch zu einer der führenden (IT-)Beratungshäuser in der Bundesrepublik aufstieg. Der Name der Gesellschaft, Integrata-Arbeitsgemeinschaft für integrierte Datenverarbeitung, war dabei von den Gründern keinesfalls zufällig gewählt. Sie wurde 1989 als ,INTErnationale Gesellschaft für Rationalisierung, Automatisierung und TechnologieAustausch“" ins Handelsregister eingetragen. Der Name brachte damit zum Ausdruck, dass sie sich - wie es für die IT-Branche damals typisch war - der rationellen Nutzung der Informationstechnologie verschrieben hatte. Den Kontakt zu dem von ihr mitgegründeten Unternehmen hielt Heidi Heilmann bis zuletzt. In der Integrata-Stiftung zur humanen Nutzung der IT von Wolfgang Heilmann engagierte sie sich von Frühjahr 2006 bis Herbst 2011 im Fachbeirat und ab dann weitere zwei Jahre im Kuratorium.

Heidi Heilmann war es bereits zu Beginn ihrer beruflichen Laufbahn wichtig, ihr Wissen und ihre Erfahrung im Rahmen von Aus- und Weiterbildung weiterzugeben, was ihren weiteren beruflichen Weg stark bestimmen sollte. Von 1967 bis 2003 war sie als Dozentin an der Württembergischen Verwaltungs- und Wirtschaftsakademie sowie an der Berufsakademie Stuttgart (Fachrichtungen Datenverarbeitung, Wirtschaftsinformatik) tätig und engagierte sich dort auch als Mitglied in verschiedenen Kommissionen zur Lehrplanentwicklung in gestaltenden Funktionen. 1973 übernahm sie für vier Jahre den Vorsitz der Fachgruppe Weiterbildung und Training im Bundesverband Deutscher Unternehmensberater. Die gewonnenen Einblicke und Erkenntnisse mögen für ihre spätere Entscheidung, hauptberuflich in Lehre und Forschung zu wechseln, ein weiterer Anstoß gewesen sein.

1980 wurde sie als Professorin für Angewandte Datenverarbeitung an die damalige Fachhochschule Furtwangen in den Fachbereich Wirtschaftsinformatik berufen. Parallel zu ihren ersten Semestern dort schloss sie 1981 ihr Promotionsverfahren an der Universität Linz zum Thema „Modelle und Methoden der Benutzermitwirkung in Mensch-Computer-Systemen“ unter Betreuung von Lutz J. Heinrich erfolgreich ab.

Heidi Heilmann gestaltete mit der für sie charakteristischen Aufgeschlossenheit für neue technische und fachliche Trends und Entwicklungen seit den Anfangsjahren der Wirtschaftsinformatik die Diskussion wesentlich mit. Sie arbeitete stets sowohl wissenschaftlich als auch praxisnah mit viel unternehmerischer Erfahrung. Sie strahlte damit nicht nur in der noch jungen Disziplin, sondern auch später, als sich das Fach etabliert hatte, Autorität aus und vermochte mit der Breite ihrer Kompetenz zu beeindrucken. Ihre an der Anwendung ausgerichteten Überlegungen, die sie, falls aus ihrer Sicht notwendig, mit einer ihr eigenen feinen Ironie zu unterlegen wusste, galten als richtungsweisend, wenn sich die aus der Elektrotechnik entwickelnde, von Männern dominierte Informatik in diesen ersten Jahren schwertat, zu verstehen, wieso es mehr als eine technische Lösung und damit eine SchnittstellenDisziplin dringend brauchte. Eine sich notwendigerweise ergebende Interdisziplinarität der Wirtschaftsinformatik durch die Rolle der Anwenderinnen und Anwender von IT-Systemen war eines ihrer zentralen Themen. 
Ein weiteres Thema, das Heidi Heilmann am Herzen lag, war das, was heute als Förderung von jungen Frauen in MINT-Fächern stark an Bedeutung gewonnen hat. Sie bedauerte oft, dass sich viel zu wenige Studentinnen für die Wirtschaftsinformatik entschieden und ließ keine Gelegenheit aus, in dieser Zielgruppe für diese wichtige und zukunftsträchtige Disziplin zu werben.

Das Jahr 1982 markierte den Beginn ihrer Tätigkeit für die HMD - Praxis der Wirtschaftsinformatik. Nach dem Tod des Gründers und Alleinherausgebers des zuvor als Loseblattsammlung erschienenen „Handbuchs der modernen Datenverarbeitung“ (HMD) übernahm sie zusammen mit weiteren Herausgebern - als einzige Frau im Gremium - die Neuausrichtung der HMD zum zentralen deutschsprachigen Journal der Wirtschaftsinformatik mit praxisnahem Wissenschaftsverständnis. Bis 2006 blieb sie verantwortliche Schriftleiterin, ehe sie das Amt an Susanne Strahringer (TU Dresden) abgab.

1985 trat sie der Gesellschaft für Informatik (GI e. V.) bei und wirkte dort als Sprecherin verschiedener Fachausschüsse und Leitungsgremien. Von 1987 bis 1989 war sie Mitglied des GI-Präsidiums.

1986 folgte sie einem Ruf der Universität Stuttgart und übernahm den Aufbau der Abteilung für Allgemeine Betriebswirtschaftslehre und Wirtschaftsinformatik des Betriebswirtschaftlichen Instituts. Wer dort technisch orientierte Betriebswirtschaftslehre studierte und sich entschloss, den Schwerpunkt Wirtschaftsinformatik zu belegen, lernte sie als ebendiese praxisnahe Professorin kennen, deren Ziel es war, ihre umfassenden Erfahrungen in Konzeption und Anwendung der IT so weiterzugeben, dass der „Praxisschock“ bei den Absolventinnen und Absolventen möglichst gering ausfiel. Sie legte dabei stets großen Wert auf klare Strukturen und eindeutige fachliche Aussagen. Konzepten, die nicht durch Beispiele zum Leben erweckt werden konnten, begegnete sie mit großer Skepsis und lehrte damit nicht nur ihre Studierenden, sondern auch ihre Doktorandinnen und Doktoranden, Sachverhalte immer und immer wieder kritisch zu hinterfragen und zu durchdenken. Wer sich auf die nicht immer einfachen fachlichen Diskussionen mit ihr einließ, erkannte rasch, dass ihre mitunter kleinteiligen Anmerkungen und ihre präzise, aber nie verletzende Kritik stets gerechtfertigt waren, was für die Qualität des Endergebnisses ohne Ausnahme einen großen Zugewinn darstellte.

Wie zuvor schon an der Fachhochschule Furtwangen mit Übernahme des dortigen Prorektorats 1983, engagierte sie sich auch an der Universität Stuttgart in der Selbstverwaltung. Von 1994 bis 1996 übernahm sie das Amt der Dekanin der Fakultät Geschichts-, Sozial- und Wirtschaftswissenschaften. Von 1997 bis zu ihrem Ruhestand im Jahr 2000 war sie gewähltes Mitglied des Senats. Dass sie mit ihrer Einschätzung hinsichtlich der Bedeutung der Wirtschaftsinformatik die Entwicklungen vorausahnte, ist sicherlich auch daran zu erkennen, dass die Universität Stuttgart nach ihrem Ausscheiden einen zweiten WI-Lehrstuhl etablierte - ein Erfolg, den sie nicht zuletzt auch ihrer Hartnäckigkeit bei den Verhandlungen hierüber zurechnete.

Ihre tiefe Überzeugung, dass Aus- und Weiterbildung sowie ein Wissenstransfer aus der Forschung in die Praxis von zentraler Bedeutung sind, veranlasste sie während ihrer Zeit an der Universität Stuttgart zur Gründung und erfolgreichen Etablie- 
rung des bis heute bestehenden „Arbeitskreis Wirtschaftsinformatik“, der mehrfach im Jahr zusammenkam und in dem Vertreterinnen und Vertreter aus Unternehmen aller Größen und Branchen in der Region über aktuelle Fragen des ,richtigen“ ITEinsatzes diskutierten.

Das Ende ihrer aktiven Zeit an der Universität Stuttgart ließ sie indes nicht ruhen. Im Jahr 2000 übernahm sie die fachliche Beratung und Herausgabe von Büchern im Bereich Wirtschaftsinformatik des dpunkt.verlags in Heidelberg. Ihre kritischen, fachkundigen und konstruktiven Hinweise sowie die überaus sorgfältige Begutachtung von Exposés und Manuskripten halfen den Autorinnen und Autoren bei der Schreibarbeit über die eine oder andere Hürde hinweg und waren ein bedeutendes Element im hohen Qualitätsanspruch des Verlags.

Die GI ehrte sie drei Jahre nach ihrem Ausscheiden aus dem aktiven Dienst an der Universität Stuttgart im Oktober 2003 durch Ernennung zum GI-Fellow. Die GIVerantwortlichen betonten die hohe Anerkennung, die sich Heidi Heilmann durch ihre wissenschaftliche Arbeit sowie ihr Engagement für die Aus- und Weiterbildung erworben hat. Gleichzeitig würdigte die GI ihren maßgeblichen Beitrag an der Entwicklung des Faches Wirtschaftsinformatik an der Universität Stuttgart und das ehrenamtliche Engagement sowohl im Präsidium der GI, als auch im Fachbereich „Informatik und Ausbildung/Didaktik der Informatik“. Ihre Mitwirkung an der Erstellung der „Neuen Empfehlungen der GI für das Informatikstudium an Fachhochschulen“ war aus Sicht der GI ein wichtiger Verdienst für die Wirtschaftsinformatik gerade mit Blick auf den Praxistransfer aus der Forschung.

Gesundheitliche Beeinträchtigungen hinderten Heidi Heilmann später, ihre Arbeit mit dem für sie bekannten hohen Anspruch fortzusetzen, was sie schweren Herzens akzeptierte.

Heidi Heilmann bleibt als profilierte Wissenschaftlerin und Lehrende in Erinnerung, aber vor allem auch als großes Vorbild für Frauen, die sich in eher männerdominierten Kontexten behaupten. Mit Professionalität und Pragmatismus war sie Vorbild darin, ihre beruflichen Pflichten mit Familienaufgaben zu vereinbaren. Heidi Heilmann starb wenige Tage vor ihrem 86. Geburtstag in Überlingen am Bodensee.

Wir, die unterzeichnenden aktiven und ehemaligen Herausgeberinnen und Herausgeber der HMD - Praxis der Wirtschaftsinformatik blicken mit Bewunderung auf das Lebenswerk von Heidi Heilmann zurück und sind dankbar für die Zeit der Zusammenarbeit. Auch wenn nicht alle heute aktiven Mitglieder sie persönlich kennengelernt haben, so ist ihr Name für uns alle doch untrennbar mit der Zeitschrift, die sie maßgeblich geprägt hat, und mit dem Fachgebiet Wirtschaftsinformatik verbunden.

Dieter Ehrenberg (em., Universität Leipzig)

Hans-Peter Fröschle (i.t.-consult, Stuttgart)

Knut Hildebrand (Hochschule Weihenstephan-Triesdorf, Freising)

Josephine Hofmann (Fraunhofer IAO, Stuttgart)

Matthias Knoll (Hochschule Darmstadt)

Andreas Meier (em., Universität Fribourg, Schweiz) 


\section{Stefan Meinhardt (SAP SE, Walldorf) \\ Michael Mörike (Integrata Stiftung, Tübingen) \\ Sara D’Onofrio (Migros Genossenschaft Zürich, Schweiz) \\ Siegfried Reich (Salzburg Research Forschungsgesellschaft, Salzburg, Österreich) \\ Stefan Reinheimer (BIK, Nürnberg) \\ Susanne Robra-Bissantz (TU Braunschweig) \\ Susanne Strahringer (TU Dresden)}

Funding Open Access funding enabled and organized by Projekt DEAL.

Open Access Dieser Artikel wird unter der Creative Commons Namensnennung 4.0 International Lizenz veröffentlicht, welche die Nutzung, Vervielfältigung, Bearbeitung, Verbreitung und Wiedergabe in jeglichem Medium und Format erlaubt, sofern Sie den/die ursprünglichen Autor(en) und die Quelle ordnungsgemäß nennen, einen Link zur Creative Commons Lizenz beifügen und angeben, ob Änderungen vorgenommen wurden.

Die in diesem Artikel enthaltenen Bilder und sonstiges Drittmaterial unterliegen ebenfalls der genannten Creative Commons Lizenz, sofern sich aus der Abbildungslegende nichts anderes ergibt. Sofern das betreffende Material nicht unter der genannten Creative Commons Lizenz steht und die betreffende Handlung nicht nach gesetzlichen Vorschriften erlaubt ist, ist für die oben aufgeführten Weiterverwendungen des Materials die Einwilligung des jeweiligen Rechteinhabers einzuholen.

Weitere Details zur Lizenz entnehmen Sie bitte der Lizenzinformation auf http://creativecommons.org/ licenses/by/4.0/deed.de. 